\title{
Experiments of cylindrical isentropic compression by ultrahigh magnetic field
}

\author{
Zhuowei $\mathrm{Gu}^{\mathrm{a}}$, Zhongyu Zhou, Chunbo Zhang, Xiaosong Tang, Yanjin Tong, Jianheng Zhao, and Chengwei Sun \\ Institute of Fluid Physics, Chinese Academy of Engineering Physics, Mianyang, Sichuan 621900, China
}

\begin{abstract}
The high Explosive Magnetic Flux Implosion Compression Generator (EMFICG) is a kind of unique high energy density dynamic technique with characters like ultrahigh pressure and low temperature rising and could be suitable as a tool of cylindrical isentropic compression. The Institute of Fluid Physics, Chinese Academy of Engineering Physics (IFP, CAEP) have developed EMFICG technique and realized cylindrical isentropic compression. In the experiments, a seed magnetic field of 5-6 Tesla were built first and compressed by a stainless steel liner which is driven by high explosive. The inner free surface velocity of sample was measured by PDV. The isentropic compression of a copper sample was verified and the isentropic pressure is over $100 \mathrm{GPa}$. The cylindrical isentropic compression process has been numerical simulated by 1D MHD code and the simulation results were compared with the experiments. Compared with the transitional X-ray flash radiograph measurement, this method will probably promote the data accuracy.
\end{abstract}

\section{Introduction}

The method of magnetic flux compression by cylindrical explosive implosion is a unique technique of ultrahigh magnetic field production [1-5]. The principle of this method is that of the conservation of magnetic flux enclosed within the boundary of a perfect conductor. Although the idea had been proposed more than half century, it is still advantaged comparing with other dynamic techniques. Besides, the magnetic pressure during the ultrahigh magnetic field production is a good tool of dynamic isentropic compression of samples. This technique will be used in many areas like high pressure physics, new material synthesizing and ultrahigh magnetic field physics [6-8].

\section{Experimental set-ups}

A schematic for the experiment set-up system is presented in Fig. 1 which includes condenser bank, the switch, a pair of coils, the liner, the explosive ring and a synchronous detonator. The initial fields within the conducting liners are produced and diffused by discharging a condenser bank through a pair of coil system at the two sides of liner. First, the condenser banks are triggered and discharged at the coils, when the discharging current is at the maximum and also the inner field is at the maximum, the cylindrical ring of explosive surrounding the liner is detonated. The subsequent implosion of the liner compresses the magnetic flux with a corresponding increasing of the magnetic field. The increasing magnetic field will exert continuous pressure on the surface of metal sample.

The capacitor bank consists of four $830-\mu \mathrm{f}, 11-\mathrm{kv}$ capacitors in all and is triggered by a semi-conductor

\footnotetext{
${ }^{a}$ Corresponding author: zhuoweigu@caep.cn
}

switch system. A pair of coils was arranged at the two sides of the liner. Each coil was organized by 16 circles of solid copper wire which were surrounded and reinforced by insulator materials. The liner was made of stainless steel and its internal diameter is $97 \mathrm{~mm}, 1.5 \mathrm{~mm}$ in thickness. The explosive used in the experiment is RDX/TNT (60/40) and $55 \mathrm{~mm}$ in thickness. A 5-6 Tesla of initial magnetic field could be obtained in the center by our facilities.

\section{Experimental results}

Some shots of experiments of EMFICG and isentropic compression have been performed. The implosion movement of the stainless steel liner was recorded by an optical turn-mirror camera and shown in Fig. 2 and the average velocity of liner is about $4-6 \mathrm{~km} / \mathrm{s}$. From a detail frame photograph of Fig. 3 the stainless steel liner will happen a serous "buckling" phenomena during the implosion process. It was caused by thin liner elasticplastic instability during the explosive driven process. In comparison a copper tube of $1 \mathrm{~mm}$ thickness was placed in the centre of the set-up and no clear "buckling" phenomena happen because of the isentropic compression.

To be emphasized, even the serious "buckling" phenomena of outside stainless steel liner will not influence the isentropic compression of inside sample because the magnetic flux inside the liner are even and the symmetrically isentropic compression of sample will also be realized.

We have carried out a 1-D hydrodynamic simulation of our experiments by a MHD code MC11D. The calculated result of liner movement and experiment result were shown in Fig. 4. From the results we could see the experiment and simulation of stainless steel liner movement are in good accord with each other.

This is an Open Access article distributed under the terms of the Creative Commons Attribution License 4.0, which permits unrestricted use, distribution, and reproduction in any medium, provided the original work is properly cited. 


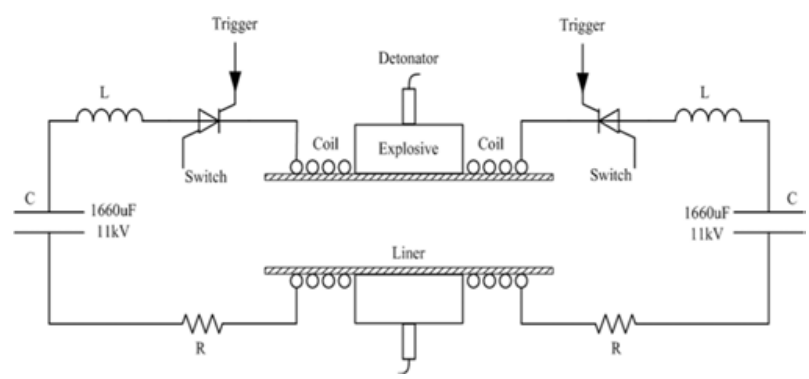

Figure 1. Schematic diagram of cylindrical implosion magnetic flux trapping system.

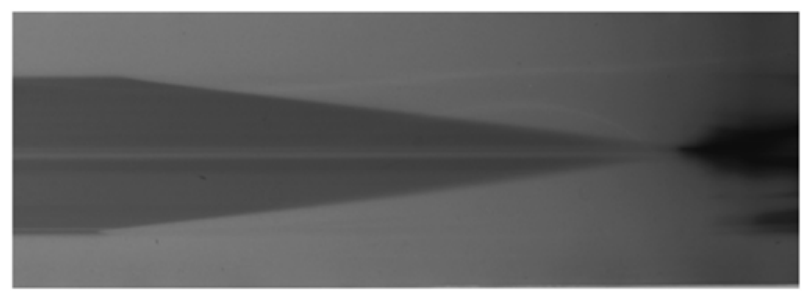

Figure 2. The liner movement recorded by optical turn-mirror camera.

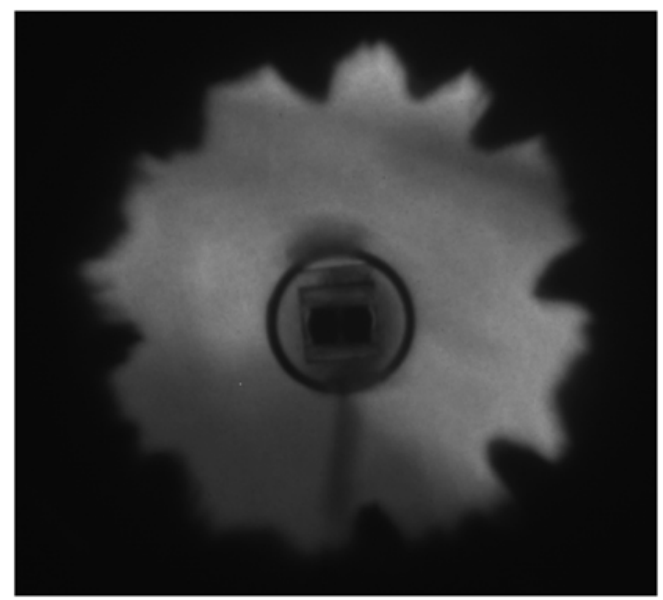

Figure 3. A copper sample was compressed by EMFICG (a PDV probe was placed inside the tube).

A typical magnetic field distribution in experiment is shown in Fig. 5 and a maximum field of about 1400 Tesla was obtained. The field measurements during the implosion were carried out by pickup coils. The $\phi 2 \mathrm{~mm}$ probe includes 4 pickup coils of $5 \mathrm{~mm}$ interval each, so we could measure the histories of magnetic field at different positions on the central axis. From the Fig. 5 we know the maximum magnetic field was obtained at the centre of set-up where a maximum compression could be realized because of the edge attenuation during the explosive driven process.

A copper tube isentropic compression experiment was carried out. In the experiment, a $\phi 11 \mathrm{~mm}$ copper tube with $2 \mathrm{~mm}$ thickness was compressed by ultrahigh magnetic field. The PDV technique was used to measure the inner free surface velocity of the tube and is shown in Fig. 6. From the experiment it is quite clear that the free surface velocity grows smoothly and the isentropic compression is verified.

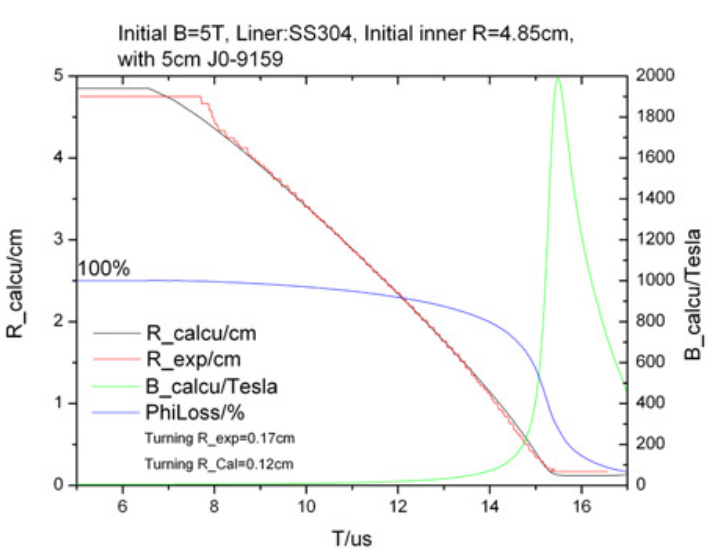

Figure 4. The simulation of liner displacement and maximum magnetic field.

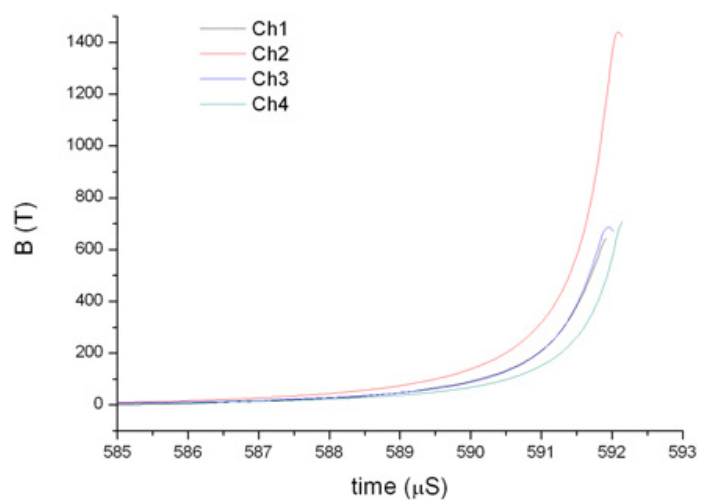

Figure 5. The magnetic field distribution in experiment $(\mathrm{Ch} 2$ is at the center of set-up).

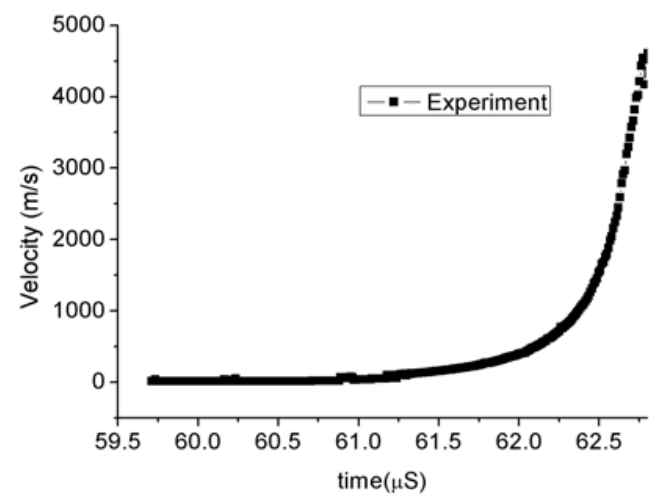

Figure 6. Inner free surface velocity of copper by isentropic compression.

\section{Analysis and conclusions}

In fact, the EMFICG technique will be very effective as a tool of isentropic compression method. Some numerical simulations have been done to show the effect of isentropic compression. In the simulation, the parameters of setup were the same with experiment, the sample was solid deuterium, and its density is $0.1766 \mathrm{~g} / \mathrm{cm}^{3}$. Two kinds of situation were considered, one is by magnetic compression, and the other is shock compression by explosive directly. 


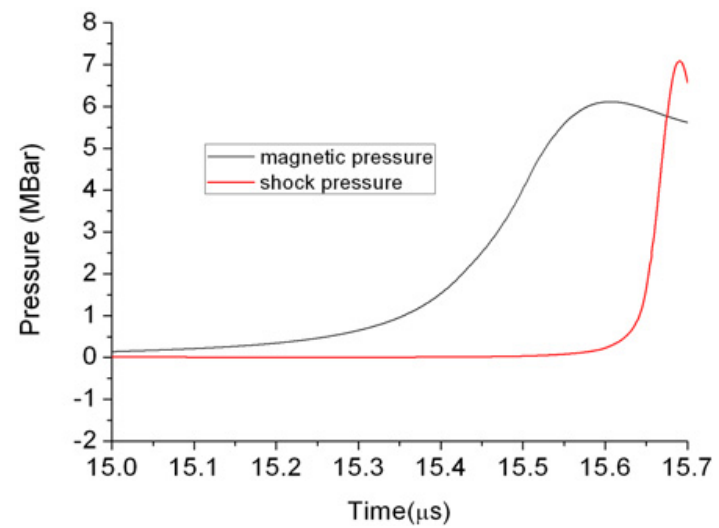

Figure 7. The pressure histories at the surface of sample.

The pressure histories on the surface of sample by magnetic compression and shock compression were shown in Fig. 7. Obviously, the magnetic compression was a kind of ramp wave loading method comparing with the shock loading. The primary calculations showed that the temperature in the sample by magnetic compression was about 10-20 times lower than that of shock situation.

Anyway, the EMFICG technique is verified by experiment and simulation as an effective isentropic compression method, and also as an ultrahigh magnetic field generator. This technique could be used widely in high pressure physics, new material synthesis and ultrahigh magnetic field physics. Some new isentropic compression experiments are under carried out and results will be published later.

\section{References}

[1] F. Herlach and H. Knoepfel, The review of scientific instruments, Vol. 36, 8 (1965).

[2] C.M. Fowler, et al., Production of very high magnetic fields by implosion, J. Appl. Phys., Vol. 31, 3 (1960).

[3] R.S. Hawke, et al., J. Appl. Phys., Vol. 43, 6 (1972).

[4] A.I. Pavlovskii, M.I.Dolotenko, N.P. Kolokolchikov, et. al., Ultrahigh magnetic fields. Physics. Techniques. Eds. V.M. Titov and G.A. Shvetsov. Moscow: Nauka. 1984. P19-22.

[5] B.A. Boyko, et. al, proceeding of the VIIIth international conference on megagauss magnetic field generation and related topics, Edited by Hans J. Schneider-Muntau, 1998, 61.

[6] R.G. Clark, proceeding of the VIIIth international conference on megagauss magnetic field generation and related topics, Edited by Hans J. SchneiderMuntau, 1998, 12.

[7] Irvin R. Lindemuth, IEEE Transactions on Plasma Science, Vol. 25, 6, 1997.

[8] G.V. Boriskov, et al. J. Low. Temp. Phys., vol. 159, 307-310, 2010. 